\title{
Cosmos and Particles: a Different View of Dark Matter
}

\author{
Leonardo Chiatti*
}

\author{
AUSL Medical Physics Laboratory; Via Enrico Fermi 15, 01100 Viterbo (Italy) and Einstein-Galilei Institute for \\ Theoretical Physics and Advanced Mathematics (I.F.M.); Via Santa Gonda 14, 59100 Prato Italy
}

\begin{abstract}
Dark matter clustered in galaxies or clusters is usually interpreted as a new type of material substance subject only to gravitational force. Alternative explanations envisage deviations of the laws of gravity, of the equations of motion or of both from their commonly accepted form. The additional possibility is explored here that the true origin of the effects depicted as "dark matter" should be seen in connection with local violations of the inertia principle, resulting from the quantized granular structure of the cosmic inertial field. Within the framework of Fantappié-Arcidiacono Projective General Relativity (PGR), this quantization appears to be the dual of that of elementary particle masses, which has been suggested for a long time. This hypothesis does not appear to contradict known facts relating to galaxy rotation and to gravitational lensing of clusters. Furthermore, it introduces a new timescale for the coupling of space expansion with structure formation, which could be of interest in cosmology.
\end{abstract}

Keywords: De Sitter relativity, dark matter, rotation curves, Mond, weak lensing.

\section{INTRODUCTION}

Dark matter is usually interpreted in three ways: as a substance, coupled solely with the force of gravity (this is probably the prevailing hypothesis, see for example ref. [1]); as a deformation of gravity (for example, in $F(R)$ gravity) [2]; as a deformation of dynamics (for example, in the MOND approach) [3]. In principle, combined approaches could also be considered.

In this article, the subject is explored from a different perspective, within Fantappié-Arcidiacono Projective Relativity. We shall assume that the reader is familiar with the language used in this approach, especially with the concept of the granular structure of "cosmic fluid" organized in "molecules". For a systematic description, please see references [4-12].

This paper is structured as follows. In Section 2 some general concepts are reviewed concerning inertia and its connections with the theories of relativity, and a possible quantization of inertia is discussed in this context. In Section 3 an attempt is made to identify hypothetical inertial field molecules with the known astronomical systems. In Section 4 connections with the weak lensing of galaxy clusters are discussed. Section 5 is dedicated to the interpretation of galactic disk rotation curves, while in Section 6 the compliance of this hypothesis with the law of Tully-Fisher is argued.

\section{GENERAL CONCEPTS}

Projective Special Relativity (PSR), perhaps more commonly known as De Sitter relativity [4-9, 13], was suggested

\footnotetext{
*Address correspondence to this author at the AUSL Medical Physics Laboratory; Via Enrico Fermi 15, 01100 Viterbo (Italy) and Einstein-Galilei Institute for Theoretical Physics and Advanced Mathematics (I.F.M.); Via Santa Gonda 14, 59100 Prato Italy; Tel: +39 761 1711055;

Fax: +39 761 1711055; E-mail: fisica1.san@asl.vt.it
}

by Fantappié in 1954 as the most general relativity that could be associated with a global covariance group of the physical laws in $(3+1)$-dimensional spacetime [14], having the Poincaré group as a local limit.

Afterwards, by applying a line of reasoning that can be traced back to Fock [15], other researchers have highlighted the connection between PSR and the inertia principle: Bacry and Levy-Leblond [16], Kerner [17], Guo et al. [18], Licata and Chiatti [9]. Roughly speaking, the global covariance group postulated by PSR, that is the De Sitter group, is the most general global covariance group which converts rectilinear uniform motions into rectilinear uniform motions; i.e. it is the most general group of global coordinate transformations which transforms inertial frames of reference into inertial frames of reference. We can say: it is the most general group of global coordinate transformations that is compatible with the inertia principle.

Projective General Relativity (PGR) is constructed starting from PSR, and consists in moving towards a more wideranging calculation of coordinates with the purpose of incorporating gravitation in spacetime metrics, i.e. in the inertial structure. Its first version was introduced by Arcidiacono in 1964 [19]. PSR and PGR are characterized by the introduction of a new fundamental constant of nature having the dimensions of a time: $t_{0}$; this is the chronological distance of a generic observer from the De Sitter horizon ${ }^{1}$. Ordinary Special Relativity (SR) and General Relativity (GR) are the limit cases of PSR and PGR, respectively, for $t_{0} \rightarrow \infty$.

\footnotetext{
${ }^{1}$ In the context of PSR, a test particle placed at a distance $x$ from a
contemporaneous observer goes away from it with a velocity $x / t_{0}$; this

${ }^{1}$ In the context of PSR, a test particle placed at a distance $x$ from a
contemporaneous observer goes away from it with a velocity $x / t_{0}$; this velocity equates the maximum speed $c$ when $x=r=c t_{0}$ (De Sitter horizon).
In PGR, the generic fundamental observer see a singularity (big bang) at a velocity equates the maximum speed $c$ when $x=r=c t_{0}$ (De Sitter horizon).
In PGR, the generic fundamental observer see a singularity (big bang) at a certain chronological distance in his past; this distance (to be not confused with the cosmic time he measures by means of a local clock) tends asymptotically to $t_{0}$.
} 
Therefore, the inertia principle constitutes the foundation of the entire relativity edifice. One naturally wonders, therefore, whether it applies without exception or if there are any limits to its applicability and, consequently, to the applicability of relativity.

From the classical theory of electrically charged elementary particles with a finite size, we know that the unconditional validity of the inertia principle no longer holds when one considers segments of particle line of universe corresponding to action increments which are less than $e^{2} / c$, where $e$ is the elementary charge and $c$ the limit velocity. This is the well-known radiation reaction problem (see ref. [20] for an elementary introduction). The lightest charged particle is the electron, thus the maximum length of this type of segment corresponds to the duration $\theta=e^{2} / m c^{3}$, as $m$ is the electron mass. This duration corresponds, apart from a non-essential $2 / 3$ factor, to the chronon introduced by Caldirola in his classical theory of the electron [21-26]. The product $c \theta$ is the classical radius of the electron and can be considered a sort of maximum spatial size of elementary particles.

It can be hypothesized that the chronon admits of the submultiples $\theta / \nu$ (where $v$ is a positive integer); the energies $E_{v}$ corresponding to these submultiples according to the uncertainty principle:

$E_{v} \frac{2 \theta}{v}=2 E_{v} v^{-1} \alpha \frac{\hbar}{m c^{2}}=\hbar$

where $\alpha$ is the fine structure constant, will correspond to an equal number of values allowed for the rest energy of particles. Letting $E_{v}=M_{v} c^{2}$, where $M_{v}$ is the rest mass of a particle, it follows from equation (1) that:

$$
M_{v}=v \frac{m}{2 a}
$$

i.e. a quantization relation for the rest mass. This result, already reported in the Fifties by Nambu [27] as an approximated empirical rule, has been reconfirmed by the recent empirical reviews by Palazzi [28], Sidhart [29, 30] and Greulich [31]. See also Chiatti [32].

In the interval $\theta$ the particle can travel a maximum spatial distance equal to $c \theta$; there is, therefore, a maximum acceleration $c \theta / \theta^{2}=c / \theta$ above which the inertia principle no longer applies unconditionally ${ }^{2}$. The breakdown of relativity at time scale less than $\theta$ could mean that the vertices of interaction among elementary particles are De Sitter microuniverses having a radius of $c \theta$. Within each of these microuniverses a microscopic version of De Sitter relativity would hold which would apply to the virtual processes, unobservable from the outside, which are involved in that interaction $[32-34]^{3}$.

Let us now turn to the cosmos. If PGR is assumed, the greatest chronological distance of an event from the here-

\footnotetext{
${ }^{2} \mathrm{By}$ this we mean that motions which violate the inertia principle imply accelerations, measured against the cosmic inertial background, greater than $c / \theta$. Let us consider, as examples of violations of the inertia principle in the classical theory of the electron, effects such as pre-accelerations, run-away solutions or the electron self-movement. The accelerations involved in these processes are normally greater than $c / \theta$.

${ }^{3}$ The concept of microuniverse has been also explored in relation with the research program on "strong gravity". See refs. $[35,36]$ for a review.
}

now (measured in Beltrami time) is $t_{0}$; projective time intervals longer than $t_{0}$ have no sense, if we refer to the past lightcone of the generic observer. On the other hand, the greatest acceleration a body can undergo in the time $t_{0}$ is $c / t_{0}$; the inertia principle could therefore no longer hold unconditionally for projective accelerations less than $c / t_{0}$. But in the local limit the projective acceleration turns into ordinary acceleration, so one can rephrase things by saying that motions involving accelerations (measured against the inertial cosmic substratum) less than $c / t_{0}$ could show deviations from the inertia principle. In other words, a free material point could show self-accelerations of the order of $c / t_{0}$.

This hypothesis can be formulated by analogy with the case of particles. It may be assumed that fundamental observers emerging from the archaic era [9-12] (mutually nonaccelerated and in accordance with a common cosmic time) are not exactly inertial. The lack of inertiality will be expressed by the field " $a$ ", where " $a$ " is the acceleration of a free body that is co-local with the observer. Please see references [9-12] for a description of the archaic era and its connection with PGR. Below we shall refer to the field " $a$ " as an "inertial field".

By analogy with equation (1) we may suppose that the quantum of energy $E_{v}$ exchanged between the field " $a$ " and matter is expressed by the relation:

$$
E_{\mathrm{v}} T_{\mathrm{v}}=E_{\mathrm{i}}\left(\mathrm{v} \alpha \frac{\hbar}{m_{d B} c^{2}}\right)=\hbar
$$

in which the mass of the electron $m$ is replaced by the "de Broglie mass" $m_{d B}=\hbar / r c$, as $r=c t_{0}$ is the De Sitter radius. The time interval:

$$
T_{\mathrm{v}}=v \alpha \frac{\hbar}{m_{d B} c^{2}}=v \alpha t_{0} ; v=0,1, \ldots, 137
$$

has this meaning: a privileged position $O$ exists where the inertia principle holds; if a free material point having null velocity (with respect to $O$ ) in a position $P$ is released, it will oscillate around the position $O$ passing through it again at time intervals $T_{v}$. Since $T_{v}$ does not depend on $O$ nor on the orientation of the segment $O P$, the field $a$ around $O$ must be spherically symmetrical with its centre in $O$. Also, $T_{v}$ does not depend on the amplitude of the oscillation, thus the oscillatory motion from $P$ to $O$ must be harmonic. This means that the material point is drawn back towards the centre of oscillation $O$ with an acceleration " $a$ " proportional to the distance $x$ from this centre.

If one supposes, in accordance with the assertions made above, that one has the greatest violation of the inertia principle for $a=c / t_{0}$, a limit value $\rho_{v}$ of $x$ will exist at which this condition will be satisfied. For $x>\rho_{v}$ one will therefore have $a=0$. We thus have:

$$
a_{\mathrm{v}}(x)=\frac{c}{t_{0}} \frac{x}{\rho_{\mathrm{v}}}
$$

for $0<x<\rho_{v}$ and $a_{v}(x)=0$ for $x>\rho_{v}$. From equations (3) and (4) the expression of the radius $\rho_{v}$ is obtained, which is quantized as a function of the index $v$, according to relation [12]: 
Table 1. Identification of Molecules

\begin{tabular}{|c|c|c|c|c|}
\hline $\boldsymbol{v}$ & $\boldsymbol{\rho}(\mathbf{k p c})$ & $\boldsymbol{T}(\mathbf{x 1 0} \mathbf{y})$ & $\mathbf{M}\left(\mathbf{x 1 0}^{\mathbf{1 0}} \mathbf{M}_{\odot}\right)$ & Possible Identification of the Molecule-Associated System \\
\hline \hline 0 & 0 & 0 & 0 & ordinary point-event where the inertia principle holds \\
\hline 1 & 6.33 & 0.111 & 8.91 & dwarf galaxies \\
\hline 2 & 25.32 & 0.222 & 142.56 & galaxies \\
\hline 3 & 56.97 & 0.333 & 721.71 & galaxies \\
\hline 4 & 101.28 & 0.444 & 2280.96 & galaxies \\
\hline 5 & 158.25 & 0.555 & 5568.75 & clusters \\
\hline 6 & 227.88 & 0.666 & 11547.36 & clusters \\
\hline 7 & 310.17 & 0.777 & 21392.91 & \\
\hline
\end{tabular}

$\rho_{\mathrm{v}}=\frac{v^{2} \alpha^{2} r}{4 \pi^{2}}$

We note that the acceleration must necessarily be directed towards the centre of oscillation and not in the opposite direction, because otherwise one would not have a finite $T_{v}$ as required by equation (3).

From equation (2) one clearly sees that in Einstein's limit $t_{0} \rightarrow \infty$ one has $E_{v} \rightarrow 0$, that is the disappearance of the field $a$, which in this limit is completely decoupled from matter and therefore loses its physical meaning. The analogy with elementary particles on which this reasoning is based is therefore only possible if a De Sitter horizon exists. In such circumstances the field " $a$ " could be null everywhere $(v=0)$, except inside 3-spherical bubbles of radius $\rho$ which we shall call "cosmic fluid molecules" [11, 12]. An ellipsoidal generalisation is perfectly possible whilst respecting the isotropy of space (if the a priori equiprobability of all possible orientations of the ellipsoid is ensured), and is treated in the Appendix. Furthermore, a number of molecules can be included one inside another, giving rise to multiperiodic motions [12]; however, only simple monoperiodic molecules will be treated in this paper. A free body located inside a molecule can stabilize in a closed orbit, with a period defined by the molecular radius and by the structure of the $a$ field within it.

\section{GENERAL CONSIDERATIONS ON COSMOLOGI- CAL EVOLUTION}

The best-fit values of $r$ and $t_{0}$ have been estimated in other works [11], with the following results:

$$
\begin{aligned}
& t_{0}=4.82 \times 10^{17} \mathrm{~s}=15.29 \times 10^{9} \mathrm{y} \\
& r=c t_{0}=15.29 \times 10^{9} \mathrm{ly}=4.69 \times 10^{9} \mathrm{pc}
\end{aligned}
$$

By applying equations (3)-(5), one thus obtains the following table of molecular radii and periods:

The null-radius molecule is nothing other than the ordinary space point where the inertia principle is exactly complied with; a free particle located in this point experiences null acceleration with respect to the local fundamental observer. These points form a continuum, which is the inertial substratum represented, in cosmological models, by the "cosmic fluid".

Non-null radius molecules form inclusions within the cosmic fluid, which correspond to regions where a free material point experiences an acceleration directed towards the centre of the molecule. Ordinary matter tends therefore to cluster in these areas, which become privileged zones for structure formation. It is as if, inside a molecule and at a distance $x$ from its centre, a probe material point were subject to the gravitational force originated by a mass $M_{\nu}(x)$ expressed by the condition:

$a_{y}(x)=\frac{c}{t_{0}} \frac{x}{\rho_{y}}=\frac{G M(x)}{x^{2}} \Rightarrow M_{v}(x)=\frac{c x^{3}}{G \rho_{v} t_{0}}$

For $x=\rho_{v}$ one obtains a total molecular mass of $8.91 \times$ $10^{10} v^{4} M_{\odot}$; this "dark mass" is set out in the fourth column of Table 1. As can easily be seen, the period $T$ is the circular revolution period. Based on the molecular radius, on the period $T$ and on the total dark mass $M$, one can attempt to identify the astronomical systems associated with the molecule. This indicative identification is set out in the last column of Table 1.

It follows from equation (6) that the dark matter inside a spherical molecule is uniformly distributed with a density:

$\mu=\frac{3 c}{4 \pi G \rho t_{0}}$

We note that if in equation (7) the molecular radius $\rho$ were replaced with the De Sitter radius $r$ one would obtain exactly the critical mean density (apart from the dilution factor $R^{3}$ caused by expansion), as discussed in reference [11]:

$$
\mu=\frac{3}{4 \pi G t_{0}^{2} R^{3}}
$$

One can easily see that the spatial mean of the field $a$, extended to volumes comprising many molecules, produces the same dynamic effects as the uniform density (8). Indeed, let us imagine a frame of reference whose origin is placed in a certain pointevent of PGR spacetime, and consider the $a(x)$ vector field seen in this reference ( $x$ is the spatial distance vector from the origin). If, $x$ being kept fixed, the frame is translated on three-dimensional space, the value of the $a(x)$ vector changes. The mean of this value on the various instantiations of the origin must be null, given the homogeneity of PGR space. The procedure can be repeated starting from a frame of reference which is rotated with respect to the preceding one, and preserving this orientation during the translation (which is certainly possible since we are dealing with 
a constant curvature space). The result must be the same because of the isotropy of PGR space. Thus, to conclude, in every frame of reference the mean value of the field $a$ in each point is zero.

A null field $a$, on the other hand, corresponds to a null density (7) and to any real-matter density whatsoever, as real matter does not affect $a$. The only acceleration which survives to the average is then the apparent acceleration directed towards the observer, which cancels the repulsion of fundamental observers caused by the hyperbolicity of primary PGR space. As we have discussed elsewhere [11], this acceleration coincides with the self-gravitation of a homogeneous distribution of matter having a density equal to the critical density, expressed by equation (8). This means that the average large-scale dynamic effect is that which would derive from a real+dark matter distribution having a homogeneous density equal to the critical one. Note that this reasoning does not resort to General Relativity and its principles.

The presence of "dark matter" grouped in molecules must have played an important role in cosmological evolution, on which at this time one can only put forward some reasonable hypotheses.

It can be believed that the field $a$, or rather a precursor, already existed in the archaic era [11, 12]. At that stage the radius of the Universe was exactly $r$ and the molecules were separate and distinct. The big bang led to a triple infinity of individual spacetimes in each of which the molecules were superimposed and merged into a very small volume, presumably that occupied by a single hadron; practically in one point. The subsequent expansion of space [and thus of the $x$ vector, argument of the $a(x)$ field] led to the unfolding of the molecules, as a consequence of the fact that the molecular radii do not undergo expansion. Gradually, the molecules, initially superimposed, separated and their separation was complete when the radius of the Universe was again equal to the de Sitter radius $r$. Starting from that moment the molecules no longer separated but simply thinned.

Primordial cosmic matter, of almost perfectly homogeneous density, gradually thickened in the molecules which were forming by separation. The molecules thus acted as condensation nuclei which trapped ordinary matter. This, by precipitating inside these nuclei with a turbulent motion, gave origin to vortices which were more or less concentric with the molecules, approximately with the same mechanisms with which a vortex forms over the drain of a tub: thus were the galaxies and clusters born. In the period between the big bang and the instant when the radius of the Universe became once again equal to $r$, the formation of structures was closely associated, therefore, with the separation of the molecules originated by the expansion of space ${ }^{4}$. At the end of this period molecule separation came to an end and the expansion became basically decoupled from structure formation. However, also after this period, molecules constitute a sort of "shelving" which imprisons ordinary matter and limits its motion [37].

\footnotetext{
${ }^{4}$ The duration of such a period in terms of kinematic time was $0.47 t_{0}$, corresponding to a period of the customary "atomic time" of $0.45 t_{0}=6.9 \times$ $10^{9} \mathrm{y}[11]$.
}

\section{MONOPERIODIC SPHERICAL MOLECULE: NEWTONIAN DESCRIPTION AND METRICS}

The acceleration $a(x)$, experienced by a free material point at a distance $x$ from the centre of a monoperiodic spherical molecule, can be imagined as derived from a potential $U$ according to the formula:

$a(x)=\operatorname{grad} U(x)=\frac{\partial U}{\partial x}$

It thus follows from equation (4) that:

$U(x)=-\frac{c x^{2}}{2 \rho t_{0}}$

for $0 \leq x \leq \rho$, and $U(x)=0$ for $x>\rho$. The acceleration is directed along the line joining the material point and the centre of the molecule, thus it follows from equation (9) that:

$v \cdot a=v \cdot \frac{d v}{d t}=\frac{1}{2} \frac{d\left(v^{2}\right)}{d t}=v \frac{d U}{d x}$

where $v$ is the velocity of the material point. Thus, if the motion of the material point is directed along the joining line:

$d\left(v^{2}\right)=2(v d t) \frac{d U}{d x}=2(d x) \frac{d U}{d x}=2 d U ;$

$v^{2}-2 U=$ constant

This latter relation expresses the principle of the conservation of energy, as can easily be seen by multiplying it by one-half of the mass of the material point. However, this relation still holds also if the mass of the point is null, i.e. it is also applicable to the case of light rays.

Let us consider then the curvature undergone by a light ray as it crosses the molecule. At the point of incidence of the ray on the molecule we have $U=0, v=c$, the speed of light in the vacuum. One thus obtains from equation (11):

$v=c\left(1-\frac{x^{2}}{r p}\right)^{1 / 2}$

This derivation is classical. The fact that the speed of light is variable is the result of the implicit assumption of the absolute synchronization of clocks; indeed, an observer in free fall towards the centre of the molecule who measures the local speed of light must find the result $c$. In other words, a covariant description is required which respects the equivalence principle. This description is provided by GR metrics in the Newtonian weak field limit:

$d s^{2}=c^{2}(1-2 \gamma) d t^{2}-(1+2 \gamma) d l^{2}$

where $\gamma=-U / c^{2}$ and the linear element $d l$ is measured in a frame of reference at rest with respect to the centre of the molecule and placed in it. In the case of light rays, one has $d s=0$ and therefore, at the first order, $c d t=(1+2 \gamma) d l$. By substituting this result into the expression of the Fermat principle $\delta \int c d t=0$ one obtains [38] the refractive index $1-$ $2 U / c^{2}$. The molecule is similar therefore to a medium having this refractive index.

It must be borne in mind that although the description provided by equation (13) is covariant and expresses the 
equivalence principle, equation (13) cannot be a solution of the General Relativity gravitational equations, for two reasons. Firstly, metrics such as (13) would imply a non-null energy tensor of matter, while equation (13) holds in the absence of matter and it is in this sense that a "dark" mass is spoken of. Secondly, equation (13) represents the metrics within the molecule, while outside of it ordinary general spacetime metrics must hold, let us say Minkowski metrics. Thus, at the molecular surface $x=\rho$ the "gravitational field" associated with the dark mass cancels out, clearly violating Gauss' theorem. This fact continues to hold true in the Newtonian approximation: equation (10) cannot be the solution of any Poisson equation.

This should not be cause for surprise, because actually we are not dealing with a genuine gravitational field but with a local violation of the inertia principle. In principle, this fact offers a first possible criterion for observational verification of the model we are suggesting. At the edge of the molecule it should be possible to observe a discontinuity of the lensing which should suddenly drop to the background value; by contrast, in models which assume a dark matter consisting of real gravitating non-baryonic matter, this leap should not be present as Gauss' theorem should apply. However, the leap can be masked both by the background lensing (if this is comparable to the leap) and by the gravitational effect of real matter which, instead, complies with Gauss' theorem. A similar discontinuity was indeed detected by Coe, Narciso, Broadhurst and Moustakas in the strong lensing data relating to the Abell 1689 cluster (A1689) [39]. The leap appears at about $300 \mathrm{kpc}$ from the centre of the cluster, and this supports an association between this object and a molecule with a quantum number $v=7$ (see Table 1). However, the situation is still difficult to interpret; an alternative explanation based on a neutrino dark matter hypothesis has recently been put forward by Nieuwenhuizen and Morandi [40].

We now wish to obtain an expression for weak lensing by a monoperiodic spherical molecule. From the general theory of weak lensing we have the following expression of the light-ray deflection angle:

$\alpha=\frac{4 G M(\xi)}{c^{2} \xi}$

where $\xi$ is the sight direction corresponding to a distance $\xi$ from the centre of the molecule and

$M(\xi)=2 \pi \int_{0}^{\xi} \sum(\xi) \xi^{\prime} d \xi^{\prime}$

In equation (15) the projected density appears:

$\Sigma(\xi)=\int \mu(\xi, z) d z$

defined as the integral, along the line of sight $\xi$, of the mass density $\mu$. The integral is executed on the intersection of the line of sight with the molecule and $z$ is the abscissa along the line of sight.

The line of sight can also be defined by means of the polar coordinates $\theta_{1}=\theta, \theta_{2}=\varphi$ with respect to the centre of the molecule $(\theta=0)$. Since the molecule is spherically symmetrical, the lensing potential $\psi$ does not depend on $\varphi$; thus, letting: $\psi_{i j}=\frac{\partial^{2} \psi}{\partial \theta_{i} \partial \theta_{j}} \quad v, j=1,2$

the only non-null term of these is $\psi_{11}$. Thus the convergence $\kappa$ and the real and imaginary parts of the shear $\left(\gamma_{1}\right.$ and $\gamma_{2}$, respectively) are expressed by:

$\kappa=\left(\psi_{11}+\psi_{22}\right) / 2=\psi_{11} / 2 ; \gamma_{1}=\left(\psi_{11}-\psi_{22}\right) / 2=\psi_{11} / 2 ;$

$\gamma_{2}=\psi_{12}=\psi_{21}=0 ; \gamma=\left(\gamma_{1}^{2}+\gamma_{2}^{2}\right)^{1 / 2}=|\kappa|$.

The reduced shear $g=\gamma /(1-\kappa)$ therefore has the value $|\kappa| /(1-\kappa)$. The convergence $\kappa$ is defined by the ratio:

$\kappa=\Sigma / \Sigma_{c r}$

where the projected critical density $\Sigma_{c r}$ is defined starting from the distances of the lens and of the lensed galaxy from the observer. This parameter is deduced from observations, as a mean across a number of lensed galaxies. In our case, therefore, weak lensing is entirely defined by $\Sigma$. Since the density $\mu$ is constant within the molecule, $\Sigma$ is simply the product of this constant by the geometric length of the intersection of the line of sight with the molecule. Since $\xi$ is always negligible compared to the distance of the lens from the observer, a simple geometrical calculation provides the relation:

$\Sigma=\frac{6 c}{4 \pi G t_{0}} \sqrt{1-\left(\frac{\xi}{\rho}\right)^{2}}$

By substituting equation (18) into equation (17), the convergence map of a spherical molecule is obtained. A word of caution is in order, however. The mass density used to deduce equation (18) is the Newtonian density (7). Thus, the deflection angle estimated with this calculation is the Newtonian one which, as is well known, is half of the Einsteinian one (which is correct). On the other hand, $\kappa$ is by definition the gradient, with respect to the line of sight, of the deflection angle $\alpha$. Thus, to produce a certain deflection angle a "Newtonian" $\kappa$ is required which is twice the "Einsteinian" one. The "Newtonian" $\kappa$ expressed by equation (18) must therefore be halved in order to provide the "Einsteinian" $\kappa$ which is the correct one. The correct formula, therefore, is:

$\Sigma=\frac{3 c}{4 \pi G t_{0}} \sqrt{1-\left(\frac{\xi}{\rho}\right)^{2}}=1.05 \times 10^{9} \sqrt{1-\left(\frac{\xi}{\rho}\right)^{2}} M_{\odot} / \mathrm{kpc}^{2}$

Finally, we note that, as immediately follows from equation (6):

$M(x)=M(\rho)\left(\frac{x}{\rho}\right)^{3}$

This formalism allows us to discuss the rather controversial case of the interacting cluster $1 \mathrm{E} 0657-558$ at $\mathrm{z}=0.296$, the famous "Bullet Cluster" [41-43]. It actually consists of a main cluster and a subclamp. The latter must have fallen inside the main cluster a long time ago and passed through it. Galaxies and dark matter, by practically penetrating each other without any mutual interaction, have left behind, in the area between the two clusters, the gas which was heated by friction and currently emits X-rays. The $\mathrm{X}$ emission area is therefore at the centre of two distinct areas of dark matter, which do not coincide with this area and can be detected 


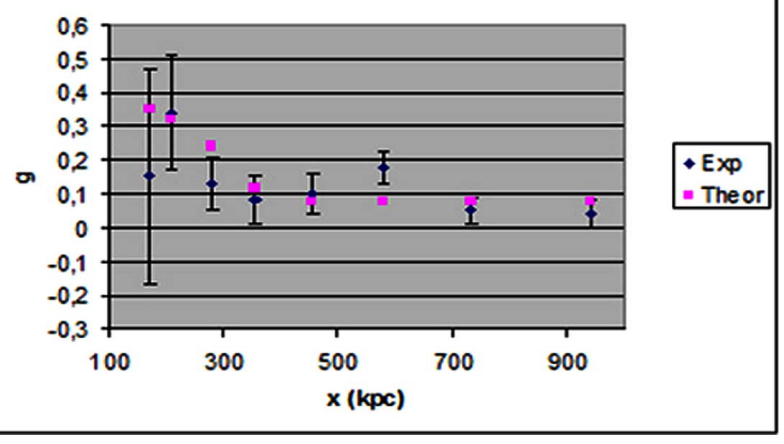

Fig. (1). Reduced shear in 1E0657-558 main cluster.

through weak lensing. According to a suggestion by Clowe, Gonzalez and Markevitch [41], which subsequently became rather popular, this arrangement supposedly proves the reality of dark matter as a material substance, in that it cannot be reproduced within the MOND formalism.

However, even if this interpretation were taken to be true, it does not eliminate the possibility that dark matter is made up of cosmic fluid molecules. The process would therefore have been the following: two galaxy clusters at the centre of two distinct molecules (probably subcomponents of a larger molecule and therefore having relative motion) supposedly collided and passed through each other. They, at the centre of their respective molecules, are now at a certain mutual distance while the gas stayed behind more or less at the place where the collision occurred.

In order to verify whether this is a tenable interpretation, we shall use equations (17) and (19) to reproduce the shear profile of the main cluster, and then compare the results with the original graphs [41]. From reference [41] we have $\Sigma_{c r}=$ $3.1 \times 10^{9} M_{\odot} / \mathrm{kpc}^{2}$. A look at the convergence maps suggests a molecular radius of $405 \mathrm{kpc}$, which corresponds to a value of $v=8$. By substituting into equations (17) and (19) we obtain:

$$
K=0.34 \sqrt{1-\left(\frac{x(k p c)}{405}\right)^{2}}
$$

as an expression of the convergence at the distance $x$ from the peak of the main cluster. In order to compare this result with the literature it is necessary to carry out the transformation $\kappa \rightarrow \lambda \kappa+(1-\lambda)$, made possible by the mass-sheet degeneracy, with $\lambda=1.07$. We obtain:

$$
K=0.36 \sqrt{1-\left(\frac{x(k p c)}{405}\right)^{2}}-0.07
$$

From equation (21) the shear profile is then derived through the relation $g=|\kappa| /(1-\kappa)$. Fig. (1) shows the graph of $g(x)$ versus the experimental data [41]; as can be seen, they agree to an acceptable degree. By substituting $x=\rho=$ $405 \mathrm{kpc}$ into equation (6), the total (dark) mass of the main cluster is obtained: $4.5 \times 10^{14} M_{\odot}$, to be compared with the observational value $M(500 \mathrm{kpc}) \approx 5 \times 10^{14} M_{\odot}$.

As regards the subclamp, examination of the convergence map suggests $v=5, \rho=158 \mathrm{kpc}, M=5.5 \times 10^{13} M_{\odot}$, in reasonable agreement with Clowe, Gonzalez and
Markevitch [41] who assume a radius of $150 \mathrm{kpc}$ and estimate a mass of approximately $7 \times 10^{13} M_{\odot}$.

\section{GALACTIC ROTATION CURVES}

The interpretation of spiral disk rotation curves undoubtedly is an important test for the ideas expressed in this paper. One immediately sees that if $a(x)$ is expressed by equation (4) the equilibrium condition on a circular orbit:

$$
\frac{v^{2}(x)}{x}=a(x)
$$

leads to rotation velocity profiles which depart from those observed. In other words, the simple expression (4) is not correct for galaxies as it would appear to be for clusters, though it is possibly still a useful starting point. Assuming equation (4), the equilibrium velocity linearly increases with the galactocentric distance $x$ reaching, at $x=\rho$, the value:

$v(\rho)=\frac{2 \pi \rho}{T}=\frac{v \alpha c}{2 \pi}=348 v \mathrm{~km} \mathrm{~s}^{-1}$

as can easily be seen from equations (3) and (5). On the other hand, these velocities are never reached in reality. Measured asymptotic velocities (inclusive therefore of the gravitational effect of ordinary matter) practically never exceed $350 \mathrm{~km} \mathrm{~s}^{-1}$; furthermore, the maximum of $a(x)$ does not correspond to $x=\rho$, but to $x=\delta$ with $\delta<<\rho$.

The (still germinal) study of empirical material suggests that the condition $v(\delta)>348 \mathrm{~km} \mathrm{~s}^{-1}$ is a pre-requisite for equation (4) to apply. For galaxies, this condition is practically never satisfied (while this is more easily so with clusters), so that some changes must be made to equation (4); such changes must return equation (4) in the special case $\delta=\rho$.

With regard to these changes, two sub-cases must be considered. If the maximum acceleration $a(\delta)$ equals $c / t_{0}$, then the following equations seem applicable:

$$
\begin{array}{ll}
a(x)=\frac{c}{t_{0}} \frac{x}{\delta} & \text { for } 0 \leq x \leq \delta \\
a(x)=\frac{c}{t_{0}} \frac{\delta}{x} & \text { for } \delta \leq x \leq \rho \\
a(x)=0 & \text { for } x>\rho
\end{array}
$$

If, instead, $a(\delta)<c / t_{0}$, observations favour the equations:

$$
\begin{array}{ll}
a(x)=\frac{c}{t_{0}} \frac{x}{\rho} & \text { for } 0 \leq x \leq \delta \\
a(x)=\frac{c}{t_{0}} \frac{\delta}{\rho} \frac{1}{1+\lambda \frac{(x-\delta)}{\rho}} & \text { for } \delta \leq x \leq \rho \\
a(x)=0 & \text { for } x>\rho
\end{array}
$$

Thus, one has two distinct classes of galaxies, according to whether equations (24) or (25) apply. Their belonging to one or the other class, however, cannot be clearly correlated with a different morphology of the rotation curves, because the gravitational effect exercised by ordinary matter constitutes an important confounding factor.

In Fig. (2) the rotation curves of six different galaxies are shown, two from the first class (Milky Way and M31) and the remainder from the second. Observational data are com- 

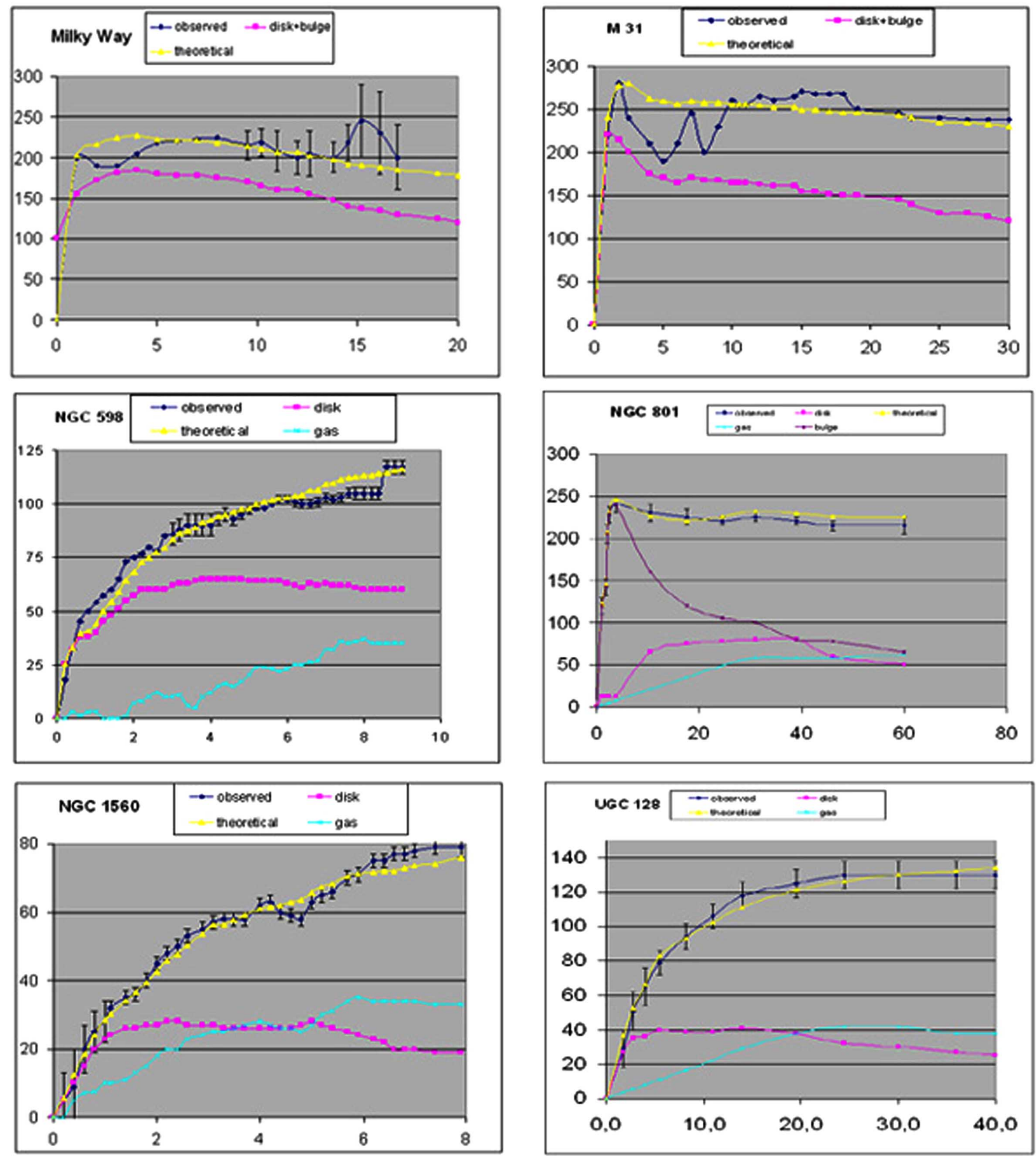

Fig. (2). Rotation curves of the six galaxies listed in Table 2. Horizontal axis is the galactocentric distance in kpc, vertical axis is the rotation velocity in $\mathrm{km} \mathrm{s}^{-1}$.

pared with the theoretical curves obtained by adding together the effects of ordinary and dark matter. The effect of dark matter is estimated, as applicable to each case, with equations (24) or (25), by adopting the parameter values set out in Table 2. In order to assess the gravitational effect of ordinary matter, photometric curves have been used for the disk or for the disk+bulge system, adding the separate contributions of the bulge and of the gas halo when available in the literature. The different components are shown in each graph.

Though these comparisons are still at an extremely preliminary stage, we think we can state that the essential features of rotation curves are properly reproduced by equations (24), (25). Of course, the problem of the theoretical justification of these equations is still an open one; they must be de- duced as particular solutions of a suitable theory of inertial field fluctuations in the archaic era.

We remark that formally equations (24), (25) can be summarized as:

$a(x)=x c^{2} \Lambda^{2}$ for $0 \leq x \leq \delta$

$a(x)=a_{\mathrm{MAX}} \delta / x^{\prime}$ for $\delta \leq x \leq \rho$

$a(x)=0$ for $x \geq \rho$,

where:

$a_{\mathrm{MAX}}=c / t_{0}, \Lambda=(r \delta)^{-1 / 2}, x^{\prime}=x$ in the first case [eq. (24)],

$a_{\mathrm{MAX}}=\left(c / t_{0}\right)(\delta / \rho), \Lambda=(r \rho)^{-1 / 2}, x^{\prime}=\delta[1+(\lambda / \rho)(x-\delta)]$ in the second case [eq. (25)] . 
Table 2. Spiral Rotation Curves

\begin{tabular}{|c|c|c|c|c|c|}
\hline Galaxy & Model & $\boldsymbol{\delta}(\mathbf{k p c})$ & $\boldsymbol{\rho}(\mathbf{k p c})$ & $\boldsymbol{\lambda}$ & $\begin{array}{c}\text { Data } \\
\text { Source }\end{array}$ \\
\hline \hline Milky Way & $(24)$ & 0.9 & & & Ref. [44] \\
\hline M31 & $(24)$ & 2.0 & & & “ \\
\hline NGC 598 & $(25)$ & 3.0 & $57(v=3)$ & 1 & Ref.[45] \\
\hline NGC 801 & $(25)$ & 10.5 & $101(v=4)$ & 4 & Ref.[46] \\
\hline NGC 1560 & $(25)$ & 3.0 & $101(v=4)$ & 1 & Ref.[45] \\
\hline UGC 128 & $(25)$ & 5.0 & $101(v=4)$ & 4 & Ref.[46] \\
\hline
\end{tabular}

If $\lambda \delta=\rho$ then $x^{\prime}=x$. When $\delta=\rho$ both the cases collapse in the harmonic oscillator [eq. (4)].

\section{COMPLIANCE WITH THE TULLY-FISHER RELATION}

In a spiral, the asymptotic circular velocity $v$ and the total luminosity $L$ are linked by the well-known Tully-Fisher relation [47]:

$$
\frac{v}{v_{0}}=\left(\frac{L}{L_{0}}\right)^{1 / \zeta}
$$

with $L_{0} \approx 1.1 \times 10^{10} L_{\odot}$ and $v_{0} \approx 220 \mathrm{~km} \mathrm{~s}^{-1}$, where $\zeta$ is between 3 and 4 . For elliptical galaxies the similar FaberJackson relation holds [48], in which the ratio in the first member is replaced by the ratio between the velocity dispersion $\sigma$ and the constant $\sigma_{0} \approx v_{0}$. Both these power laws are compatible with the interpretation of dark matter presented in this article.

Let us consider the equilibrium condition:

$$
\frac{v^{2}(R)}{R}=G \frac{M(R)}{R^{2}} \Rightarrow v^{2}=\frac{G M(R)}{R}
$$

in which $R$ represents the radius of the luminous disk and $v=$ $v(R)$. In this relation, $M(R)$ naturally includes both the contribution of ordinary matter and that of dark matter [obviously, $M(R)$ represents the true mass included in the radius $R$ only in the case of spherical symmetry]. At this point we must distinguish between the galaxies for which equations (24) are valid from those for which equations (25) are valid.

For galaxies of the first class, the velocity $v$ increases in a linear manner in the interval $x<\delta$, after which it stays constant [eq. (24)]. If the initial climb were solely determined by dark matter we would have:

$$
\frac{v^{2}}{\delta}=\frac{c}{t_{0}} \text {. }
$$

We can take into account the effect of ordinary matter by multiplying the second member of this relation by $\beta>1$. One therefore has:

$$
\frac{v^{2}}{\delta}=\frac{c}{t_{0}} \beta
$$

If $v$ remains constant for $\delta<x<\rho$ also in the presence of ordinary matter, one has:
$M(R)=\frac{R}{G} \frac{c}{t_{0}} \delta \beta=\frac{R^{2}}{G} \frac{c}{t_{0}} k \beta$,

in which it has been assumed that $\delta=k R$. Thus:

$R=\sqrt{\frac{G t_{0}}{c k \beta} M(R)} \Rightarrow v^{2}=\frac{c}{t_{0}} k \beta=\sqrt{\frac{c k \beta G}{t_{0}} M(R)}$.

I.e.:

$v=\sqrt[4]{\frac{c k \beta G}{t_{0}} M(R)}$

Therefore, in a population of galaxies having homogeneous values of the indices $k$ and $\beta$, then $v \propto M^{1 / 4}$. On the other hand, it is a known fact that for galaxies there is a precise mass-luminosity ratio, whereby the last relation becomes $v \propto$ $L^{1 / 4}$. One obtains, therefore, for this population, equation (26) with $\zeta=4$.

Let us now examine the galaxies of the second group. For these, the maximum acceleration is not $c / t_{0}$, but $\left(c / t_{0}\right)(\delta / \rho)$. The factor $\beta$ will now be estimated at $x=R$ rather than at $x=$ $\delta$. With this redefinition we have:

$$
\begin{aligned}
& \frac{v^{2}(R)}{R}=\frac{c}{t_{0}} \frac{\delta}{\rho} \beta \frac{1}{1+\lambda \frac{(R-\delta)}{\rho}} \approx \frac{c}{t_{0}} \frac{\delta}{\rho} \beta \Rightarrow v^{2}=\frac{c \beta \delta R}{\rho t_{0}}=\frac{c k \beta}{\rho t_{0}} R^{2} \\
& M(R)=\frac{R v^{2}}{G}=\frac{c k \beta}{G \rho t_{0}} R^{3} \Rightarrow R=\sqrt[3]{\frac{G \rho t_{0}}{c k \beta} M(R)} \\
& v^{2}=\frac{c k \beta}{\rho t_{0}}\left(\sqrt[3]{\frac{G \rho t_{0}}{c k \beta} M(R)}\right)^{2} \\
& v=\sqrt[3]{G\left(\frac{c k \beta}{\rho t_{0}}\right)^{1 / 2} M}
\end{aligned}
$$

Thus for a galaxy population having homogeneous values for the indices $k$ and $\beta, v \propto M^{1 / 3}$. With the same arguments set forth for the first class of galaxies, one therefore has equation (26) with $\zeta=3$.

In both cases the logic of relation (26) is clear: the luminosity is linked to $R$ by power laws, and at the same time to $M$ by the mass-luminosity ratio; thus $M$ defines $R$, and since $k$ is given, $\delta$ is defined. $\delta$ in turn defines (for a given radius $\rho)$ the maximum acceleration resulting from dark matter alone, and the consequent equilibrium velocity. From this, as a result of $\beta$, one obtains the asymptotic velocity expressed by equation (26).

Equations (28) and (29) could in principle be used to test the model, if the uncertainties on the mass were small. By way of example, let us consider a small-sized elliptical galaxy for which it can be hypothesized that $v=1, k \approx 1$ and $\beta$ $\approx 1$. For this type of galaxy, an indicative value of $10 M_{\odot} / L_{\odot}$ may be assumed for the mass-luminosity ratio. Based on the reasoning set forth above, it seems consistent to classify this galaxy among those of the first group and thus to apply equation (28) to it.

By substituting, therefore, in equation (28), $M$ with (10 $\left.M_{\odot} / L_{\odot}\right) L$ and letting $L=L_{0}=1.1 \times 10^{10} L_{\odot}$ one obtains $v=$ $305 \mathrm{~km} \mathrm{~s}^{-1}$. If we remember that, for elliptical galaxies, the velocity dispersion $\sigma$ is linked to $v$ by the relation $\sigma=v / 2^{1 / 2}$ 
and that in equation (26) the velocities in the first member must be replaced by the related dispersions (Faber-Jackson relation), one obtains, at the denominator of the left member, $\sigma=\sigma_{0}=v_{0}=216 \mathrm{~km} \mathrm{~s}^{-1}$. This value practically coincides with the often-adopted one of $220 \mathrm{~km} \mathrm{~s}^{-1}$, and this constitutes a clue in support of the hypothesis that the cosmological parameter $t_{0}$ governs the dynamics of galaxies through the acceleration $c / t_{0}$.

\section{CONCLUSIONS}

The hypothesis presented in this paper can be summarized by stating the existence of a universal acceleration field. In morphological terms, such a field would appear null everywhere except in some spherically shaped "islands". A material point placed within one of these islands would be attracted towards the centre by an acceleration vector having a modulus less than or equal to $c / t_{0}$.

These "islands", which we have called "molecules", of the "cosmic fluid" representing the continuum of fundamental observers, were formed in a pre-big bang era. This is a suggestive term to say that they were virtual fluctuations of the inertial field which were present in the "pre-vacuum" state, subsequently emptied of energy by the big bang. These fluctuations have survived in a frozen state and have constituted the privileged condensation nuclei of ordinary matter, thus contributing in an important way to the formation of the structures.

That which we see today as dark matter which has clustered in the structures (galaxies and clusters) is in effect the manifestation of the acceleration field inside the molecules where the structures condensed. Systems such as the Bullet Cluster, from this point of view, do not prove the substantial nature of dark matter (at least in the same sense in which hydrogen and neutrinos are considered "substances"), and they are fully compatible with the hypothesis of interacting clusters associated with different sub-molecules in relative motion within the same mother molecule. Naturally, it can be maintained that the inertial field is a form of matter and that in this sense dark matter is actually a substance.

This hypothesis complies with the equivalence principle (unlike other hypotheses such as the MOND approach) and a "metric" description of the molecules is therefore possible. However, the gravitational equations are not applicable to this description, because ordinary matter - though it undergoes the effect of the field - is not its source. The field, therefore, is not coupled with any energy tensor or similar source term; it consists in a pure spacetime curvature (if we privilege the metric description). The problem of determining a change to the gravitational equations that is capable of including the molecules is still an open one for the time being.

If the hypothesis is correct, the inertia principle can be subject to limitations defined by the fundamental constants $\theta$ and $t_{0}$. It is interesting to note that these two constants are linked by the relation $\theta / t_{0} \approx 2^{-1 / \alpha}$ [49-51], which involves the fine structure constant. The nature of the relation between this constant and inertia is still entirely unexplored. Generally speaking, it can be said that the violation of the law of inertia on the elementary particle scale (associated with the scale parameter $c \theta$ ) is connected with the granular structure of ordinary matter, that is to the existence of elementary par- ticles and to the origin of their mass. Instead, the violation of this same law on a cosmic scale (defined by the scale parameter $c t_{0}$ ) is associated with the granular structure of the acceleration field, and is the root of dark matter.

\section{CONFLICT OF INTEREST}

None declared.

\section{ACKNOWLEDGMENTS}

I wish to thank Prof. Ignazio Licata for many useful discussions and Prof. Christian Corda for his suggestions and the revision of manuscript.

\section{APPENDIX}

It is possible that cosmic fluid molecules have a more general geometry than the spherical one. This is actually what ought to take place when three independent harmonic oscillators act on a generic test material point. The equations of motion of this point will thus be:

$$
\ddot{x}=-\omega_{x}^{2} x ; \quad \ddot{y}=-\omega_{y}^{2} y ; \quad \ddot{z}=-\omega_{z}^{2} z ;
$$

where:

$\omega_{x}=\sqrt{\frac{c}{t_{0} A}} ; \omega_{y}=\sqrt{\frac{c}{t_{0} B}} ; \omega_{z}=\sqrt{\frac{c}{t_{0} C}}$

From the quantization rules:

$p_{x}=\mathrm{v}_{x} \alpha t_{0} ; p_{y}=\mathrm{v}_{y} \alpha t_{0} ; p_{z}=\mathrm{v}_{z} \alpha t_{0}$

and from the relations $\omega_{x}=2 \pi / p_{x}$ etc. one obtains the relations:

$A=\frac{v_{x}^{2} \alpha^{2} r}{4 \pi^{2}} ; \quad B=\frac{v_{y}^{2} \alpha^{2} r}{4 \pi^{2}} ; \quad C=\frac{v_{z}^{2} \alpha^{2} r}{4 \pi^{2}}$.

The acceleration modulus is expressed by:

$a=\left(\ddot{x}^{2}+\ddot{y}^{2}+\ddot{z}^{2}\right)^{1 / 2}=\frac{c \sigma}{t_{0}}$

with

$\sigma=\sqrt{\left(\frac{x}{A}\right)^{2}+\left(\frac{y}{B}\right)^{2}+\left(\frac{z}{C}\right)^{2}}$

The surfaces on which this modulus assumes the same value are the ellipsoids $\sigma=$ constant; the modulus assumes the extreme value $c / t_{0}$ on the ellipsoid $\sigma=1$, which thus represents the outer limit of the molecule. The molecule is therefore an ellipsoid, with three generally different axes. The isotropy of space thus requires that there be no privileged orientation of the normal axes of the molecule.

The indices $v_{x}, v_{y}, v_{z}$, are positive integers and therefore periodic orbits certainly exist for a probe material point. Indicating their lowest common multiple with $v_{0}$, the revolution period of the material point will be:

$$
p=v_{0} \alpha t_{0} \text {. }
$$

\section{REFERENCES}

[1] Brook C, Kawata D, Gibson B, Freeman K. The emergence of the thick disk in a cold dark matter Universe. Astro phys J. 2004; 612: 894-99. 
[2] Corda C. Interferometric Detection of Gravitational Waves: the Definitive Test for General Relativity. Int J Mod Phys 2009; D18: 2275-82.

[3] Bekenstein J, Milgrom M. Does the missing mass problem signal the breakdown of newtonian gravity? Astro Phys J 1984; 286: 7-14.

[4] Cacciatori S, Gorini V, Kamenshchik A. Special relativity in the $21^{\text {st }}$ century. Annalen der Physik 2008; 17 (9-10): 728-68.

[5] Arcidiacono G. Projective relativity, cosmology and gravitation. Nonantum: Hadronic Press 1986.

[6] Licata I. Universe without singularities; a group approach to de sitter cosmology. Electr J Theor Phys 2006; 3(10): 211-24.

[7] Benedetto E. Fantappié-Arcidiacono spacetime and its consequences in quantum cosmology. Int $\mathrm{J}$ Theor Phys 2009; 48 (6): 1603-21.

[8] Benedetto E. Lie group of spacetime. ISRN Astron Astrophy 2011, ID 873830, doi:10.5402/2011/873830.

[9] Licata I, Chiatti L. The archaic Universe: big bang, cosmological term and the quantum origin of time. Int J Theor Phys 2009; 48: 1003-18.

[10] Licata I, Chiatti L. Archaic universe and cosmological model: bigbang as nucleation by vacuum. Int J Theor Phys 2010; 49: 2379402 .

[11] Chiatti L. De Sitter relativity and cosmological principle. Open Astron J 2011; 4: 27-37.

[12] Chiatti L. A possible mechanism for the origin of Inertia in De Sitter-Fantappié-Arcidiacono projective relativity. Electr $\mathbf{J}$ Theor Phys 2012; 9(26): 11-26.

[13] Chiatti L. The fundamental equations of point, fluid and wave dynamics in the De Sitter-. Fantappié-Arcidiacono projective special relativity. Electr J Theor Phys 2010; 7: 259-80.

[14] Fantappié L. Su una Nuova Teoria di Relatività Finale. Rend Accad Lincei 1954; 17: 158.

[15] Fock VA. The theory of space, time and gravitation. London: Pergamon Press 1964.

[16] Bacry H, Lévy-Leblond JM. Possible Kinematics. J Math Phys 1968; 9(10): 1605-15.

[17] Kerner HE. An extension of the concept of inertial frame and of lorentz transformation. Proc Natl Acad Sci USA 1976; 73: 141821.

[18] Guo HY, Huang CG, Xu Z, Zhou B. On special relativity with cosmological constant. Phys Lett A 2004; 331: 1-7.

[19] Arcidiacono G. Spazi di cartan e teorie unitarie. Collectanea Mathematica 1964; 16: 149-68.

[20] Feynman RP. Lectures on Physics (vol. II chapt. 28). London: Addison-Wesley 1970.

[21] Caldirola P. A new model of classical electron. Nuovo Cim Suppl 1956; 3: 297.

[22] Caldirola P. On the introduction of a fundamental interval of time in quantum mechanics. Nuovo Cim Lett 1976; 5(16): 151-5.

[23] Caldirola P, Casati G, Prosperetti A. On the classical theory of the electron. Nuovo Cim 1978; 43 A: 127-42.

[24] Caldirola P. On a model of the relativistic electron deriving from the introduction of the chronon. Nuovo Cim Lett 1978; 23: 83-8.

[25] Caldirola P. The chronon in the quantum theory of the electron and the existence of the heavy leptons. Nuovo Cim 1978; 45A(4): 54979.

[26] Caldirola P. On a relativistic model of the electron. Nuovo Cim 1979; 49A(4): 497-511.

[27] Nambu Y. An empirical mass spectrum of elementary particles. Prog Theor Phys 1952; 7: 595-6.
[28] Palazzi P. Available at: http://www.particlez.org/p3a. Files 2004001-v2, 2004-002-v1，2005-001-v2，2005-002-v1，2005-003-v1, 2005-005-v1, 2006-001-v1, 2006-002-v1.

[29] Sidhart BG. A formula for the mass spectrum of baryons and Mesons. arXiv:physics/0306010v1.

[30] Sidhart BG. A QCD Generated Mass Spectrum. arXiv:physics/0309037v1.

[31] Greulich KO. Calculation of the masses of all fundamental elementary particles with an accuracy of approx. $1 \%$. J Mod Phys 2010; 1: 300-2.

[32] Chiatti L. A possible model for the mass spectrum of elementary particles. arXiv:physics/0905.3794; accepted for publication.

[33] Caldirola P. Introduzione del Cronone nella Teoria Relativistica dell' Elettrone. De Finis F, Pantaleo M, Eds. Centenario di Einstein. Firenze: Giunti Barbera 1979.

[34] Caldirola P. Progressi nella Teoria dell' Elettrone. Annuario EST Mondadori 1979; 65-72

[35] Recami E. Confinement and hadron-hadron interactions by general relativistic methods. Prog Part Nucl Phys 1982; 8: 401-411.

[36] Recami E, Hernandez F, Kretly LC, Barbero P. Elementary particles as Microuniverses. Rev Bull Calc Math Soc 1998; 6: 67-72.

[37] Peebles PJE, Nusser A. Nearby Galaxies as pointers to a better theory of cosmic evolution. Nature 2010; 465: 565-9.

[38] Levi-Civita T. Fondamenti di meccanica relativistica. Bologna: Zanichelli 1928.

[39] Coe D, Narciso B, Broadhurst T, Moustakas L. A high-resolution mass map of galaxy cluster substructure: lens perfect analysis of A1689. Astrophys J 2010; 723: 1678-1702.

[40] Nieuwenhuizen TM, Morandi A. Prediction for the neutrino mass in the KATRIN experiment from lensing by the Galaxy Cluster A1689. J Cosmology 2011.

[41] Clowe D, Gonzalez A, Markevitch M. Weak lensing mass reconstruction of the interacting cluster 1E 0657-558: Direct evidence for the existence of dark matter. arxiv:astro-ph/0312273.

[42] Markevitch M, Gonzalez AH, Clowe D, et al. Direct Constraints on the Dark Matter Self-Interaction Cross-Section from the Merging Galaxy Cluster 1E 0657-56. arxiv:astro-ph/0309903.

[43] Clowe D, Bradac M, Gonzalez AH, et al. A direct empirical proof of the existence of dark matter. arxiv:astro-ph/0608407.

[44] Klypkin A, Zhao H, Somerville RS. LCDM-Based Models for the Milky Way and M31 I: Dynamical Models. Astrophys J 2002; 573 : 597-613.

[45] Moffat J. Reinventing gravity. Talk given at Astronomy and Space Exploration Society-Faces of Space, University of Toronto, 01-142010. Available at Moffat website: http://www.johnw-moffat.com/ papers.html

[46] Sanders RH. The published extended rotation curves of spiral galaxies; confrontation with modified dynamics. Astrophys J 1996; 473: 117 .

[47] Tully RB, Fisher JR. A new method of determining distances to Galaxies. Astron Astrophys 1977; 54: 661-73.

[48] Faber SM, Jackson RE. Velocity dispersions and mass-to-light ratios for elliptical Galaxies. Ap J 1976; 204: 668-78.

[49] Sternglass EJ. A model for the early Universe and the connection between gravitation and the quantum nature of matter. Nuovo Cim Lett 1984; 41(6): 203-8.

[50] Sternglass EJ. The quantum condition and the non-euclidean nature of space-time. Ann New York Acad Sci 1986; 480: 614-7.

[51] Sternglass EJ. Before the Big Bang: the origins of the Universe. New York: Four Walls Eight Windows Publisher 1998. 